Aus der chirurgischen Universitätsklinik zu Zürich. (Direktor: Prof. Dr. F. Sa u e r bruch.)

\title{
Ein Fall von cystischer Dilatation des vesikalen Ureterendes.
}

Von Dr. Th. Verriotis.

(Mit 4 Abbildungen.)

Die cystische Erweiterung des vesikalen Ureterendes konnte bis zu der Zeit, wo man dic Cystoskopie noch nicht kannte, nur durch die Autopsie festgestellt werden und wurde als embryologische Mißbildung beschricben. Während des Lebens war eine Diagnose wegen der vielseitigen, meist unklaren Symptome nicht nöglich. Die Krankheit blieb denn auch den Klinikern lange Zeit hindurch unbekannt. Erst seit Desormaux mit Hilfe seines Endoskops und $\mathrm{Nitz}$ e durch seine geniale Erfindung des Cystoskops das Innere der Blase der direkten Besichtigung zugänglich gemacht hatte, konnte eine Diagnose auch während des Lebens gestellt werden. G roB glick war der erste, welcher im Jahre 1899 mittels der cystoskopischen Untersuchung einen Tumor in der Harnblase diagnostizicrte. Bei der Operation fand er jedoch statt des Tumors eine cystische Erweiterung des Ureters vor. Garrè stellte mit Hilfe des Cystoskops im Jahre I902 als erster eine sichere Diagnose und operierte seine Kranken mit gutem Erfolge. Über die Pathogenese jedoch und über gewisse Fragen, welche die Art der einzuschlagenden Therapie betreffen, ist die Diskussion noch nicht abgeschlossen, besonders weil die Ätiologic sehr mannigfaltig ist und es sich bis jetzt bei der Seltenheit der Krankheitsfälle nur um einzelne Publikationen handelt.

Der folgende Fall soll zur Ätiologie und Symptomatologie dieser Erkrankung einen Beitrag bilden. 


\section{Krankengeschichte.}

Der 2Ijährige Patient ist Schreiner von Beruf. Er stammt aus gesunder Familie und erkrankte im Alter von sechs Jahren an einer Lungenentzündung mit anschließendem Empyem. Einen operativen Eingriff am Thorax überstand er gut und hatte seither keine Beschwerden mehr.

Vor etwa $I^{1 / 2}$ Jahren verspürte Patient zum erstenmal nach Beendigung des Urinierens einen leichten Schmerz, welcher einige Sekunden andauerte. Patient mußte zu dieser Zeit häufig urinieren, besonders beim Radfahren. Nach 3-4 Monaten wurde das Urinieren immer frequenter und die Schmerzen dauerten während der ganzen Zeit des Harnlassens an. Zu jener Zeit bekam Patient plötzlich nach einem Frühstück heftige Schmerzen im Unterleib mit Harndrang, ohne jedoch Wasser lösen zu können. Die Schmerzen dauerten etwa $z$ wei Stunden. Dadurch, daß Patient durch eine heftige Bewegung seinen Körper in eine andere Lage brachte, hörten plötzlich die Schmerzen auf und Patient konnte sofort wieder urinieren. Vier Monate später bekam Patient einen ähnlichen Anfall, der denselben eben beschriebenen Verlauf nahm: heftige Körperbewegung, plötzliches Auftreten der Schmerzen, Möglichkeit des Urinierens.

Diese Anfälle wiederholten sich nicht mehr. Aber die eingangs erwähnten Beschwerden blieben zurück und nahmen stets bei der Fahrt im Eisenbahnzuge und beim Radfahren zu. Hämaturie will Patient nie beobachtet haben, will auch nie geschlechtskrank gewesen sein. Da mit verschiedener Diät und allen möglichen Arzneien keine Besserung eintrat, kam Patient zu uns in die chirurgische Klinik.

Patient ist ein großer, kräftig gebauter junger Mann, von frischem, gesundem Aussehen. Die Untersuchung der verschiedenen Organe ergibt nichts Pathologisches. Blasengegend nicht schmerzhaft. Genitalien gut entwickelt. Hoden und Nebenhoden mit Samenstrang in normaler Lage und von normaler Beschaffenheit. Beiderseits keine auffallenden Größenunterschiede. Prostata ist etwas vergrößert, sonst weich, glatt und symmetrisch gebaut. Samenblasen nicht zu fühlen. Harnröhre zeigt beim Abtasten keine Verhärtung, läßt eine 24-Charrière-Sonde glatt durch. Kein Residualurin in der Blase. Die Blasenkapazität beträgt ca. $700 \mathrm{ccm}$. Die Untersuchung auf Steine ist negatv.

Urinuntersuchung. Der Urin ist klar, reagiert schwach sauer und hat ein spezifisches Gewicht von 1028. Spuren von Eiweiß, kein Zucker.

Mikroskopische Untersuchung: Viele polynukleäre Leukocyten und einige Plattenepithelien. Zylinder oder Nierenepithelien sind nicht vorhanden. Tuberkelbazillen nicht nachweisbar, nur einige grampositive Diplokokken.

Nach der Krankengeschichte und der klinischen Untersuchung 
zu urteilen, leidet der Patient an ciner Cystitis von unbekannter Ursache. Hinsichtlich der Anfälle und der gleichzeitigen Harnverhaltung könnte man an einen Fremdkörper, z. B. einen Stein der Blase denken. Dagegen spricht jedoch der Umstand, daß der Patient nie Blut im Urin gehabt hat und die Untersuchung auf Steine negativ ausgefallen ist. Einen Tumor kann man ebenfalls ausschließen im Hinblick auf die Anamnese und das Fehlen von Hämaturien.

Unter diesen Umständen führten wir die Cystokopie aus, die uns die gewünschte Aufklärung brachte.

Cystoskopische Untersuchung (siehe Fig. I, 2, 3): Das Cystoskop läßt sich leicht in die Blase einführen. Diese wird mit $300 \mathrm{ccm}$ Borwasser gefüllt. Das T rig on u $\mathrm{m} \mathrm{L}$ i e u ta u d ist leicht injziiert. Die linke Urctermündung ist in normaler Lage und besitzt normale Form. An Stelle der rechten Uretermündung sieht man einen taubeneigroßen Tumor, wclcher in dic Blase vorspringt. Der Tumor ist glatt, mit Schleimhaut bedeckt, von normaler Vaskularisation. An der oberen Tumorfläche bemerkt man cine Rinne, deren untere Lippe sich über die obere legt. Sie läßt einon $1 / 3 \mathrm{~cm}$ langen Schlitz vermuten (Fig. I).

Am rechten Rand des Tumors bemerkt man das Vorhandensein eines Oedema bullosum (Fig. 2). Bei längerem Betrachten sieht man, daß der Tumor eine große Beweglichkeit besitzt und daß sein Volumen periodisch wechselt in fo'gender Weise: Der Tumor dehnt sich wie ein Ballon aus, indem er nach vorn gegen das Orificium urethrac internum zu sich verlïngert. In diesem Moment ist der Tumor durch. sichtig und seine Wandungen sind sehr gespannt. Nach einigen Sekunden sieht man gewisse leichte Kontraktionen seiner Wände. Es ergießt sich dann ein kurzdaucrnder Strom aus der erwähnten Rinne, welcher das optische Feld des Instrumentes trübt. Der Tumor nimmt dann an Größe al) und wcicht gleichzeitig nach hinten zurück. Damit kommt er an dic Stelle zu liegen, wo eigentlich die Ausmündungsstelle des rechten Urcters scin sollte und faillt in melhreren Falten zusammen.

Diesc Erscheinung wiederholt sich in Abschnitten von 15 bis 25 Sekunden. Oberhalb des Tumors sieht man eine schräg nach außen verlaufende, bleistiftdicke. $3^{-5} \mathrm{~cm}$ lange Wölbung, die mit dem Tumor in Verbindung steht. Sowohl die Wölbung, als auch der Tumor schwcllen an, jedoch in der Weise, dal3 man den Übergang dieser Schwellung von der Wölbung zum Tumor erkennen kann. Bei einem solchen Ergebnis kann man mit Sicherheit die Diagnose auf eine cystische Erweitcrung des rechten endovesi$\mathrm{k}$ alen Ureterendes stellen.

Beim Vergleich der Urinentleerung aus den beiden Ureteren sieht man einen großen Unterschied zwischen dem dilaticrten Ureter und dem normalen. Während dic Entleerung des normalen Ureters. 

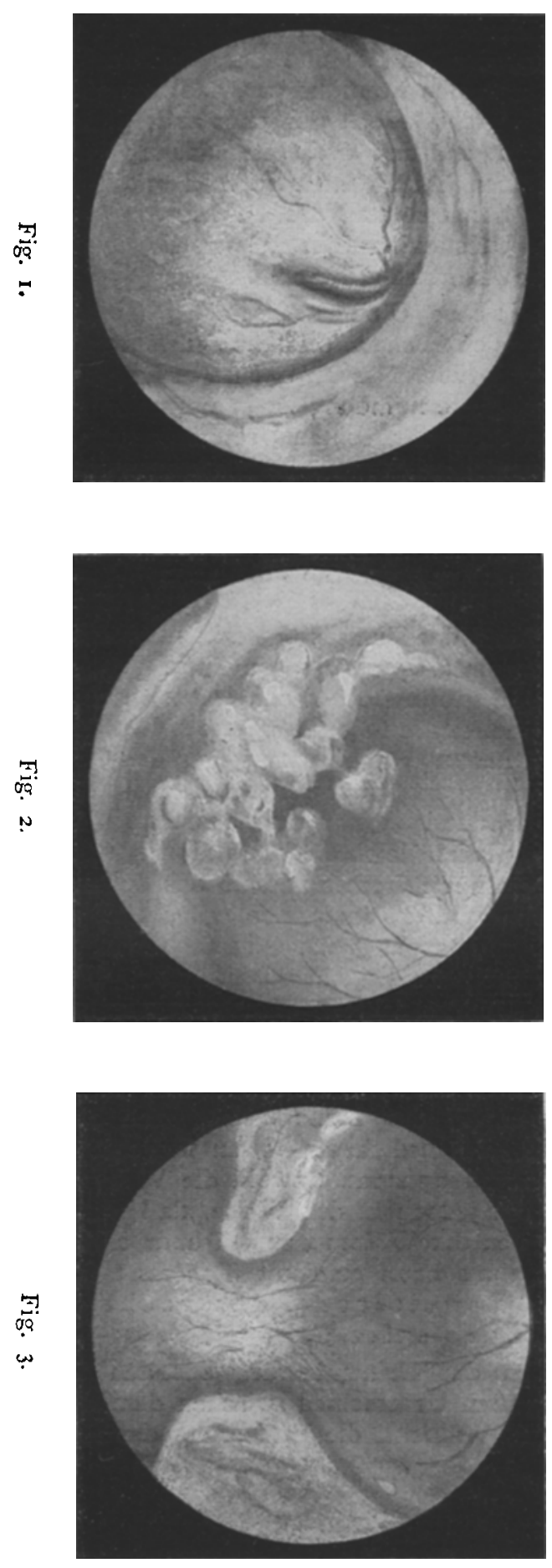
strahlenartig in Abschnitten ron 50 Sekunden erfolgt, ist die des dilatierten mehr diffus und folgt in Abschnitten ron $I_{5} 25$ Sekunden in bedeutend größeren Mengen. Der Ureterenkatheterismus gelingt beiderseits. Ein in den dilaticrten [reter eingefühtter Katheter (Größe 6) kann bis zum Nierenbecken vorgeschoben werden. Man stellt fest, daß etwa $70 \mathrm{ccm}$ Residualurin herauskommen. In den linken Creter wird ein Katheter (Größe 6) eingeführt und gleichfalls bis zum Nierenbecken vorgeschoben.

Die Nicrenbeckenkapazität ergibt Rechts 80 ccm. l.inks $7 \mathrm{~cm}$.

I) Nie Nenfunktionsprüfung wird vorgenommen.

Rechts:

Menge während einer Stunde.

$60 \mathrm{ccm}$

lìweib

Zucker

Harnstoff

Chloride

Indigocarmin nach ro Minuten.
I. in ks:

$100 \mathrm{ccm}$

negativ

negativ

1,90 Proz.

1,22

nach 4 Minuten.

Nach den oben crwähnten Untersuchungen konnte kein Zweifel bestehen, daB die Ursache der Beschwerden unseres Patienten in der cystischen Dilatation des rechten Ureterendes lag. P'rof. Sauerbruch entschloß sich deshalb zur operativen Entfernung der Cyste.

Operationsbericht: 22. II. 19I8. Operateur l'rof. Sa uerbruch.

Nach Jodalkoholdesinfektion wird in Äthernarkose die Sectio alta ausgeführt. Bei dersclben wird der cystoskopische Befund bestätigt. Der Lircterwulst erscheint jetzt nur weniger gefüllt und kleiner als bei der Cystoskopie (walnußgroß). Ein kleiner, fingerdicker, nach Anzichen der Cyste sichtbarer Wulst, verläuft schräg nach oben und auswärts, dem rechten Ureter entsprechend. Es wird jetzt dieser cystische Tumor durch Umschneiden von vorn und von der Seite her mobilisiert. Nach Umschneidung dieses ganzen Tumors vom Innern des Kegels aus wird dic Umsäumungsnaht vorgenommen, so daß ein breiter Fingang in den rechten Ureter zustande kommt. Die Blutung steht. Dic Blase wird zweischichtig nach Mikulicz geschlossen. Weichteilnaht unter Einlegen eines feines Gazestreifens. Dauerkatheter eingelegt.

Der postoperative Verlauf war die ersten Tage sehr gut. Der Lrin wurde nach 3 Tagen klar und der Daucrkatheter entfernt. Nach + Tagen aber trat eine Bronchitis ein und die Temperatur stieg auf 38,5. Der Urin blieb klar, ohne pathologische Bestandteile. Die Miktion war beschwerdefrei. Bald darauf Rückgang der Temperatur auf normale Höhe. Patient verließ nach drei Wochen das Bett be. schwerdefrei, mit normaler Miktion und normalem Urinbebefund. 
Bei der vor der Entlassung vorgenommenen Kontrollcystoskopie sieht man in der sonst normal erscheinenden Blase an Stelle der früheren Cyste jetzt ein langes, ovales Loch, dessen Ränder mit Schleimhaut bedeckt sind. Die Urinejakulation ist kräftig, aber ohne ausgeprägte Kontraktion der Lippen des neuen Ostiums. Der Ureterenkatheterismus ergibt beiderseits keine Nierenbeckenretention.

$\mathrm{P}$ a t h o g e n e se: Die Pathogenese dieser cystischen Dilatation des Ureterendes hat zu verschiedenen Diskussionen Anlal3 gegeben. Die meisten Autoren nehmen als Grundursache eine mehr oder weniger starke Stenose des Ureterostiums an, die eine kongenitale oder erworbene sein kann. Bei der kongenitalen handelt es sich um Mißbildungen im Urogenitalapparat, die die Zahl der Ureteren oder ihre Ausmündungsstelle betrifft. Bei Ureterverdoppelungen, welche nach $\mathrm{S} \mathrm{chewkuneko} 2-4$ Proz. aller Sektionen ausmachen, kann der eine von vornherein völlig blind endende Ureter eine Retentionscyste bilden (Lechler, Streubel, Sinnreich, Elliesen) oder am vesikalen Ende fast bis zur vollständigen Obliteration verengt, langsam sich cystisch dilatieren. Ebenso bei der kongenital abnorm tiefen Mündung des Harnleiters, wobei das Ostium entweder im Bereich des Sphinkter vesicae ( $\mathrm{Cohn}$ ) oder sogar distal von demselben (Bostroem I, Geerdt und Haushalter, Johnson) in den Samenwegen enden kann.

Die e rworbene Stenosierung des Ostiums kann in erster Linie durch entzündliche Prozesse verursacht werden, die sich in der Uretermündung abspielen. Sie können vom Ureter selbst ausgehen, oder von der Blase, der Niere, dem Nierenbecken oder von andern Organen der Nachbarschaft auf den Ureter übergehen. Wir glauben aber, daß die Stenosierung des Ostiums allein nicht genügt, um eine Dilatation des Ureters zu verursachen. Wir wissen aus klinischer Erfahrung, daß bei Patienten mit mechanischem Hindernis am Ureterostium (Ureterstein oder entzündliche Stenosierung des Ureterendes, Blasentuberkulose) keine Dilatation des endovesicalen Ureterendes zustande kommt, wohl aber eine Hydro- oder Pyonephrose. Die Arbeiten von Albarran und Halle, welche experimentell durch Verengerung des Ureter. orificiums eine Hydronephrose bei Tieren erzeugen wollten, zeigen, daß die Dilatation des Ureters extravesikal zustande kommt und nicht intravesikal. Auch bei einer Anzahl von Patienten mit dieser 
cystischen Dilatation des Ureterendes stellen wir fest, daß keine wesentliche Verengerung des Ureterostiums vorhanden ist (E $11 \mathrm{~s}$, unser Fall usw.). Es handelt sich vielmehr um einen anormalen Verlauf des endovesikalen Teiles des Ureters unter der Blasenschleimhaut. Wie schon aus den Arbeiten von Disse und $\mathrm{K} \mathrm{r}$ a u s e bekannt ist, verliert sich der Ureter innerhalb der Blasenwand nicht in deren Bestandteilen, sondern bleibt ganz selbständig und steckt in einer Lücke der Blasenmuskulatur. Nur seine Schleimhaut, das Epithel und die bindegewebige Unterlage setzen sich direkt in die Schleimhaut der Blase am Orificium ureteris fort. Da der Ureter in schiefer Richtung auf die Blasenwand trifft und innerhalb derselben seine Richtung nicht ändert, so endigt er mit kürzerem oberen und längerem unteren Rand. Von den Muskeln der Blase ist die Ureterwandmuskulatur durch eine Bindegewebslage getrennt und sie zeichnet sich vor ihr durch ihre viel geringere Dicke aus. Nach dem Durchtritt durch die Muskularis der Blase gelangt der Ureter als selbständiges, dickwandiges Rohr in die Submucosa der Blase und verläuft dann zwischen Blasenepithel und Blasenmuskulatur, nach $\mathrm{Kr}$ a use durchschnittlich I2-I $4 \mathrm{~mm}$ lang, bis zur Einmündungsstelle. Das untere Ende des Ureters wird von einem rohrartigen Fortsatze der Blasenmuskulatur umgeben (Ureterscheide Waldeyers). Sie ist durch lockeres Bindegewebe mit der Wand des Ureters rerbunden.

Wenn jedoch der Harnleiter die Muskulatur nicht schräg, wie das die Norm ist, sondern in gerader Richtung durchbohrt, um dafür in größerer Ausdehnung submukös weiter zu verlaufen, so haben die Kontraktionen der Muskulatur der Blasenwand, die auf den normalen Harnleiter einen Druck ausüben und eine Erweiterung desselben nicht zustande kommen lassen (Bostroem), auf diesen terminalen Teil keinen Einfluß mehr. Hinzu kommt, daß dieses submuköse Ureterende viel nachgiebiger sein muß, weil es nicht mehr rings von Blasenmuskulatur umgeben ist. Bei unscrem Falle konnten wir bei der Cystoskopie wie auch bei der Operation feststellen, daß das endovesikale, hinter der Cyste bleistiftdicke, $3-4 \mathrm{~cm}$ lange Stïck des Ureters unter der Blasenschleimhaut verlief und dilatiert war. Unter diesen anatomischen Verhältnissen ist es erklärlich, daß diese Partie des Ureters im 
Falle, wo eine kongenitale oder erworbene Stenose seines Orificiums vorkommt, dilatiert wird. Aurh cine nur temporär be-

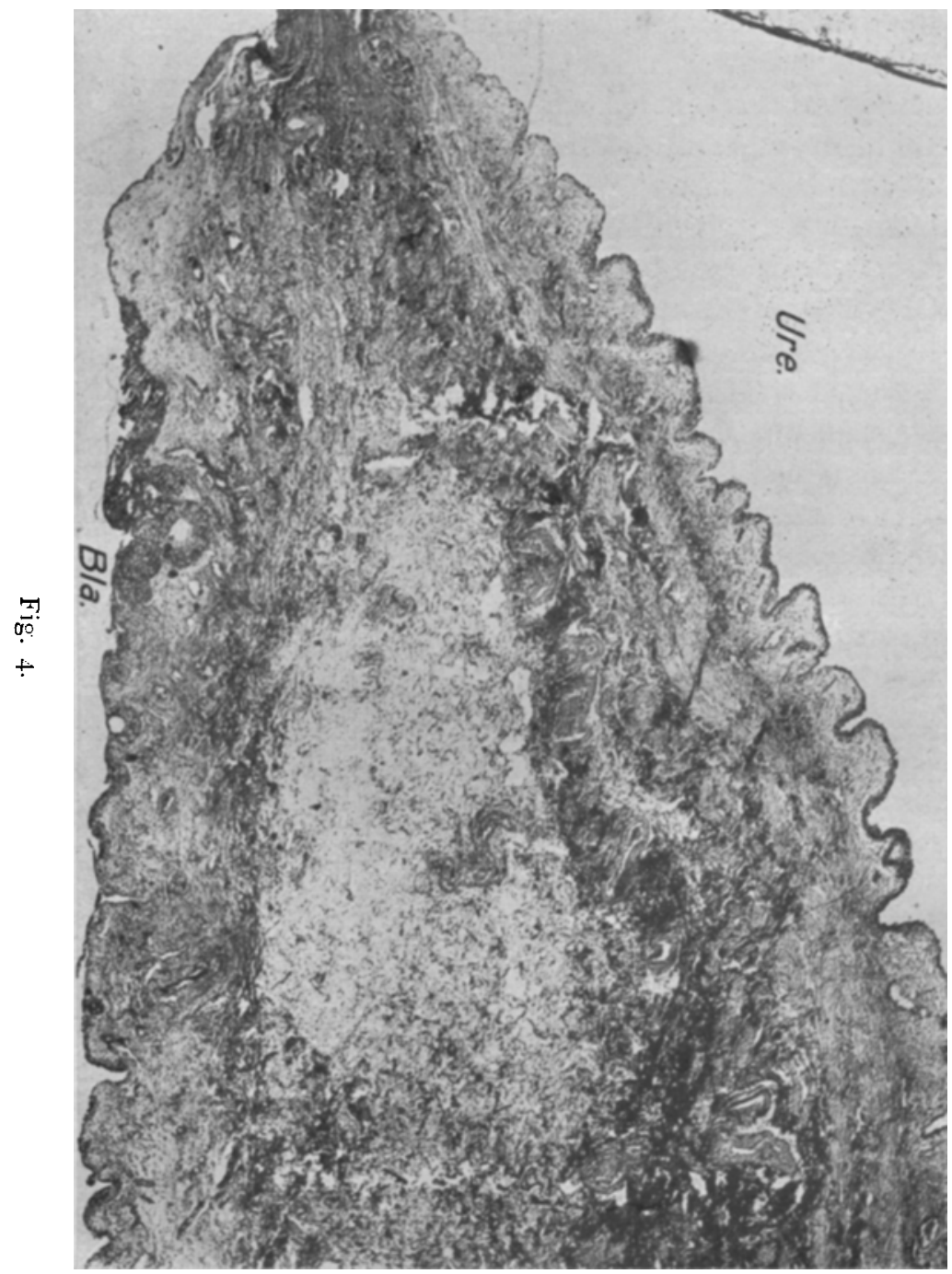

stehende Stenose des Orificiums, wie sie durch eine akute Lintzündung verursacht wird, kann auf Grund der beschriebenen Anomalien den endovesikalen Teil des Ureters zur Dilatation bringen. 
Bei der histologischen Untersuchung (Fig. 4) unseres Falles ergab sich, daß der uretrale Teil der Cyste bedeckt ist mit einer Epithelschicht, unter welcher wir glatte Muskulatur erkennen, dic in 3 Schichten angeordnet ist, und zwar können wir eine innere Längslage, einc mittlere kräftige, zirkuläre und eine diskontinuierliche äußere Muskelschicht unterscheiden. Die vesikale Fläche der Cyste ist ebenfalls mit einer Epithe'schicht bedeckt. lhre Unterlage bildet jedoch nur Bindegewebe, Blasenmuskulatur läl3t sich nicht erkennen. Unsere $\Lambda$ uffassung über die Natur der cystischen Dilatation des Ureterendes ist also kurz zusammengefaßt so, daß auf Grund einer anatomischen Anomalie (langer submuköser Verlauf des Ureterendes) durch eine dauernde oder vorübergehende Stenosierung des Orificiums eine Erweiterung des intravesikalen Ireterendes zustande kommt.

Symptomatologie: Was die Symptomatologie betrifft, kann man sagen, daß kein pathognomonisches Symptom der Krankheit besteht. Die Symptome sind sehr mannigfaltig, atypisch und verschieden. Das läßt sich erklären durch die Größe des 'Tumors, durch seine Beweglichkeit, durch die Iinge oder Breite des cystisch erweiterten Ureters und besonders durch die begleitcnden sekundären Schädigungen seitens des Harnapparates, welche eine uberwiegende Rolle in der Symptomatologie aller bis jetzt beobachteten Fälle spiclen. Mehr oder weniger große Säcke, deren Ostien nicht verengt und deren Entleerung so gut wie unbehindert waren, konnten deshalb symptomlos bleiben und als Nebenbefund entdeckt werden (Toebben II, Sankott). Schmith und Rämi, Elliessen dachten an einen Blasenkatarrh, da der Patient nur einen heftigen Harndrang als Symptom hatte. Nelsen glaubte an cine Blasengeschwulst, Bostroem an eine Hydronephrose, $\mathrm{Engl}$ ish an Prostatahypertrophic, (; $\mathrm{r} \cap \beta \mathrm{glick}$ an Nierenkolik. In denjenigen Fällen aber, in welchen die Folgezustände der cystischen Dilatation, wie Pyonephrose und Nephritis, zu schweren allgemeinen Erscheinungen geführt hatten und eine sichere Lokalisierung nicht mehr möglich war, hat man an cine Meningitis tuberculosa (Toebben II), Bronchopneumonie (Wranny) oder diffuse Peritonitis ( $\mathrm{F} \mathrm{unke}$ ) gedacht. Die schmerzhaften Anfälle mit Harnverhaltung finden wir bei den Fällen von Cohn, Els I und in unserem Falle. 
In dem von $\mathrm{E} 1 \mathrm{~s}$ beschriebenen Falle war eine ständige Behinderung des Harnlassens vorhanden. „Als bei dem Patienten die quälenden Tenesmen und die Unmöglichkeit, den Urin spontan zu entleeren, sich einstellten, d. h. die Harnröhrenöffnung durch die großen Cysten verlegt wurde, verstand er sich dadurch zu helfen, daß er - unbewußt allerdings - die Druckverhältnisse in der Blase $z u$ beeinflussen und ein zu großes Minus harnröhrenwärts zu verhindern suchte. Dadurch, daß er vor bzw. im Beginn der Miktion die Harnröhre nahe der Eichel zusammendrückte, erreichte er einerseits, daß mit Öffnung des Sphincter internus in der Harnröhre gleicher Druck wie in der Blase bestand, also nicht so stark harnröhrenwärts getrieben wurde." Bei unserem Patienten aber war zweimal anfallsweise komplette Harnverhaltung mit heftigen Schmerzen aufgetreten, die als Folge der Einklemmung der Cyste in das Orificium urethrae internum anzusehen ist. Bei den selbständigen peristaltischen Bewegungen des dilatierten Ureters, welche unabhängig von den Blasenkontraktionen erfolgen, bewegte sich der dilatierte Harnleiter vor dem Orificium int, urethrae hin und her. Befand er sich aber gerade einmal zufällig während einer Blasenkontraktion vor der inneren Harnröhrenmündung, so wurde er eingeklemmt, was Blasenverschluß zur Folge hatte. Diese Einklemmung konnte eine Zeitlang bestehen, bis der immer reichlicher sich ansammelnde Harn die Blase soweit ausdehnte, da $B$ das eingeklemmte Ureterende von der Urethra weggezogen wurde. Andernfalls kann aber die krampfartige Kontraktion der Blase den cystisch dilatierten Ureter so fest einklemmen, daß ein Urinabfluß verunmöglicht wird und eine Nierenkolik entsteht. Wir glauben, daß nur durch eine Nierenkolik die heftigen Schmerzen, die unser Patient bei den beiden Anfällen gehabt hat, erklärt werden können. Bei einer zweistündigen Retention beginnen die Schmerzen nicht so plötzlich und sind nicht so heftig, wie sie unser Patient gehabt hat. Durch eine brüske Beugung seines Körpers nach hinten erfolgte eine gewisse Dehnung des Ureters und sein eingeklemmter Teil schlüpfte heraus und ging in die ursprüngliche Lage zurück. Damit diese Erscheinung zustande kommen kann, muß der cystische Ureter eine genügende Größe erreichen, um sich bis zum Orificium urethrae internum ausdehnen zu können, oder er 
mul3 ganz anormal in der Nähe des Blasenhalses ausmünden. Bei l'atienten weiblichen Geschlechts prolabiert der cystische Ureter, so dals man ihn aus der Vulva heraustreten sehen kann (Knoop, Haushalter et Jaques, Hiller, Johnson). Hämaturien können auch vorkommen. Sie finden ihre Erklärung in einer chronischen Hyperämie des Blasenhalses, des 'Trigonum und auch der Ureterschleimhaut selbst (König, Fehnwich). Außerdem kann das Vorhandensein von Steinen in dem dilatierten Sacke eine Hämaturie verursachen ( $\mathrm{Els}$ ).

Di a g nose: Die einzige Methode zur Erlangung einer sicheren Diagnose ist die Cystoskopie, wie denn auch die in der Literatur bekannten Fälle auf diese Weise diagnostiziert wurden. Mittels der Cystoskopie bekommen wir nicht nur einen guten Iinblick in die auslösenden Ursachen der Symptome seitens der Harnblase, sondern auch in die Begleiterscheinungen und sekundär erfolgenden Schädigungen der Niere. Auf solche Untersuchungen hin kann man erst eine entsprechende Behandlung vornehmen, dic aber stets auf den vorliegenden Fall abgestellt sein muß.

Bei der cystoskopischen Untersuchung stellen wir in einem solchen Falle folgendes fest:

I. Is handelt sich um einen oder zwei Tumoren von wechselnder Größe, bohnen- bis mandarinengroß, die mit normaler Blasenschleimhaut bedeckt und von glatter Oberfläche sind. In den Fällen, wo eine Cystitis vorliegt, bemerkt man mitunter, daß die Oberfläche des Tumors ein Oedema bullosum erkennen läßt, wie in unserem Falle, oder Farbenveränderungen und stärkere Vaskularisation der Blasenwandung.

2. I)as Volumen des Tumors ist wechselnd, ändert sich periodisch, bleibt aber konstant, wenn die Wände infiltriert oder hart sind.

3. Der Tumor ist mehr oder weniger durchsichtig, je nach der Beschaffenheit seiner Wände.

4. Zwischen dem Beginne der Anschwellung des Tumors und seiner Wiederannahme ist je nach dem Falle eine Trübung des optischen Feldes oder ein fadenförmiger, klarer Harnstrom wahrzunehmen, der aus dem Tumor herausfließt. Die ureterale Ejakulation kann klar oder trübe sein. 
5. Während der Anschwellung sinkt der Tumor gegen das Orificium urethrae int., zieht sich aber, indem er sich entleert, gleich wieder zurück.

6. Seine Beziehung zum Ureter läßt sich auf folgende, Weise bestimmen: Wenn man die korrespondierenden Ausmündungsstellen der Ureteren aufsucht, beobachtet man, daß der Tumor mit einem mehr oder weniger langen und breiten Stiel an der eigentlichen Mündungsstelle des Ureters der Blasenschleimhaut aufsitzt und in den meisten Fällen an seinem oberen Teile eine kleine, leicht rötliche Öffnung erkennen läßt.

7. Die verschiedenen Lageänderungen des Patienten, wie der wechselnde Füllungsgrad der Harnblase, können das Vo. lumen des Tumors beeinflussen.

Prognose: Wie man aus der Literatur ersehen kann, sind die anatomischen Beziehungen und die verschiedenen erworbenen und angeborenen Schädigungen und Mißbildungen, welche die Krankheit begleiten, sehr mannigfaltig und von Fall zu Fall verschieden. Hiervon hängt ganz und gar die Prognose ab.

In gewissen Fällen ist einige Tage nach der Geburt der Tod

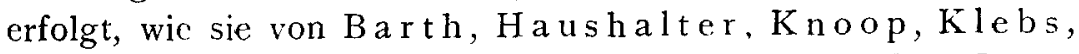
Hibler I und II, Johnson u. a. beschrieben wurden. In anderen Fällen haben die Kranken trotz häufiger Harnbeschwerden ein längeres Leben erreicht. Die Autopsie erst ergab bei ihnen Schädigungen der Nieren, wie Pyonephrose. Hydronephrose usw. (Smith, Remy, Elliesen, Xavier, Delose, Carrell u. a.). Bei anderen Patienten ist die Krankheit erst in einem Lebensalter von $62^{--76}$ Jahren erkannt worden $(\mathrm{B} \mathrm{u} \mathrm{r} \mathrm{c} \mathrm{khardt}$, Sankott, Tanlly, T,ilienfeld, GroBglick).

In allgemeinen kann man sagen, daß bei gut erhaltener Funktion der Nieren und rechtzeitiger Diagnose eine erfolgreiche Behandlung durchgeführt werden kann.

Die Therapie, die angegeben wurde, war verschieden. Ga r r è führte bei seinem Patienten 1902 die totale Resektion der Cyste aus, und zwar in folgender Weise: Nach Eröffnung der Blase resezierte er die in die Blase vorsprpringende Cyste und vernähte die zwei Schleimhautränder der Blase und der Ureter. Andere 
Autoren haben diese Naht nicht gemacht und die Epithelisierung erfolgte sehr schnell; clas Orificium blieb breit offen. Horraison, Klose, Cohn, Bunge haben auf endovesikalem Wege mit einem Messer oder schcrenförmigen Instrument das Orificium der Cyste breit gespalten, so daß eine große Öfnung entstand. Außerdem hat Bunge für sehr ängstliche Patienten die Dilaticrung des Orificiums durch steigernde Bougierung vorgeschlagen.

Wir glauben, daß die oben erwähnten Methoden gute Resul tate geben können, wenn sie bei entsprechend geeigneten Fällen angewendet werden. Welche Methode, ob die nach Sectio alta oder die auf endovesikalem Wege vorzuzichen sei, hängt vom vorliegenden Falle ab. Jede hat ihre bestimmten Indikationen, je nach dem Zustand der oberen Harnwege, die zusammengefaßt folgende sind:

Bei denjenigen Patienten, deren Nierenfunktion eine gute und deren Allgemeinzustand befriedigend ist, wo aber die klinische und cystoskopische Untersuchung eine starke chronische Cystitis ergibt, die Cyste cin großes Volumen und indurierte und starr bleibende Wände hat, glauben wir, daß die totale Entfernung der Cyste nach Sectio alta indiziert ist. Es bleibt dann die Blase nach der Sectio alta und Drainierung in Ruhe, während auch bei genügender Erweiterung des Ostiums (durch endovesikale Osteotomie) dic starren Wände der (yste bestehen bleiben und diese als ein Fremdkörper in der Blase wirken würde.

Ganz anders liegen die Verhältnisse bei den Patienten, wo die Nierenfunktion eine schlechte ist. Eine Narkose kann auf den renalen Apparat solcher Patienten eincn ungünstigen Einfluß haben, da er an sich schon geschädigt ist. Unter solchen Umständen oder bei Fällen, wo das Volumen der Cyste eine mittlere Größe hat und ihre Wände dünn sind und sich unter dem Cystoskop als kontraktil erweisen, glauben wir, daß die Behandlung auf intravesikalem Wege zum Ziele führen kann. Selbstverständlich verlangen dicse intravesikalen Operationen eine spezielle Ubung; deshalb finden sich, trotz der immer ausgezeichneten Erfolge, wenige Fälle in der Literatur.

Die hyperkonservative Behandlung der steigernden Dila- 
I54 VerRioris, Ein Fall von cystischer Dilatation des vesikalen Ureterendes.

tation durch Bougieren des cystisch erweiterten Ureters können wir nur bei den Patienten empfehlen, die sich um keinen Preis zur Operation verstehen wollen. Sie bleibt immer eine palliative Behandlung, die nur temporäre Resultate ergeben wird, wie bei den Urethralstrikturen.

Schließlich muß man bei Fällen, wo die sekundären Störungen der Niere (Pyonephrose) sehr fortgeschritten sind, in erster Linie die Behandlung dieser sekundären Störungen ins Auge fassen und diejenige der Cyste erst in zweiter Linie. 